\title{
SCINTILLATING FIBRE TRACKER FRONT-END ELECTRONICS FOR LHCB UPGRADE
}

Albert Comerma*, on behalf of LHCb Scintillating Fibre Tracker Group

*Physikalisches Institut - Heidelberg Universität,

Im Neuenheimer Feld 226, 69120 Heidelberg, Germany

e-mail:comerma@physi . uni-heidelberg • de

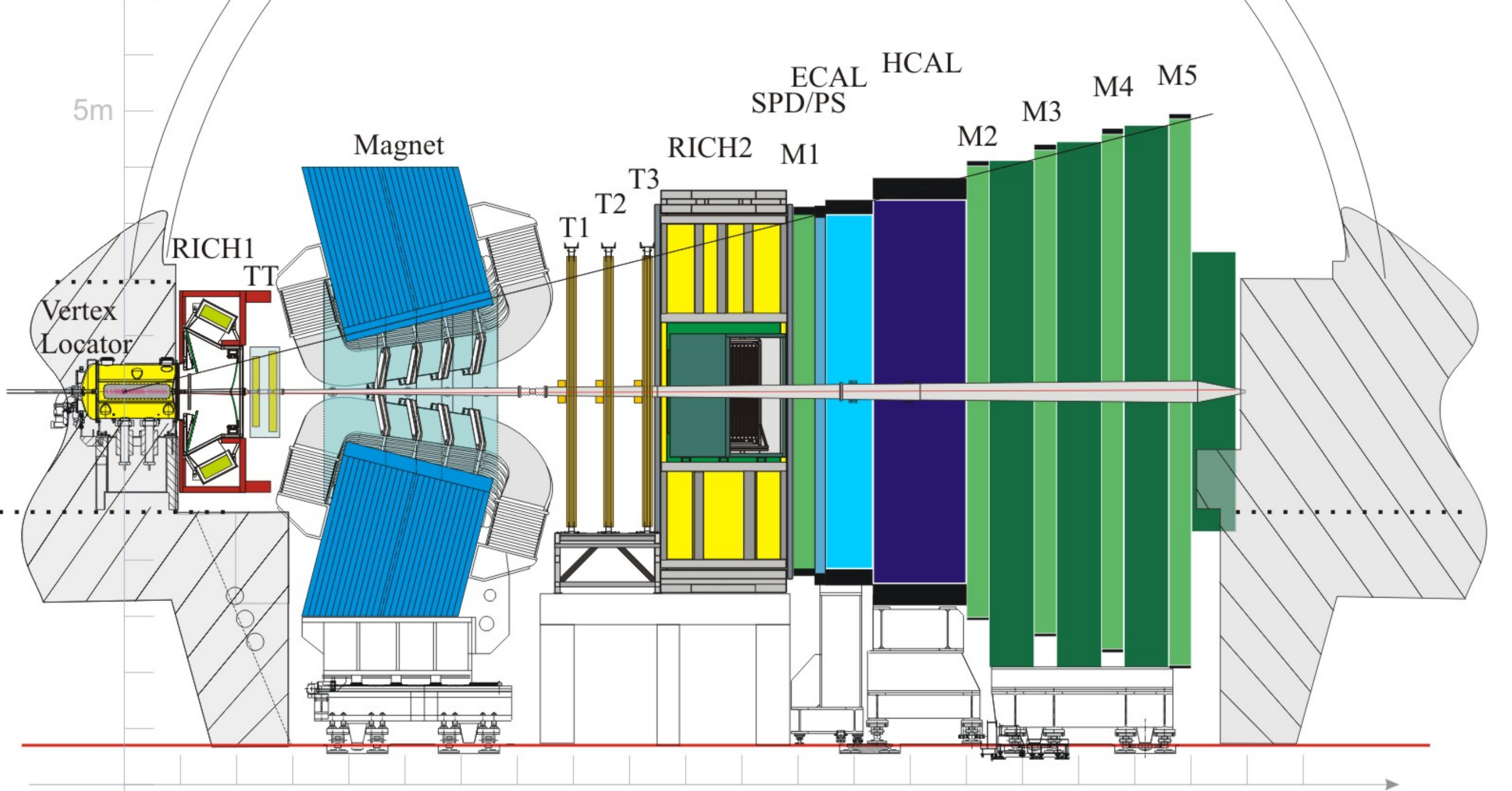

LHCb experiment cross section

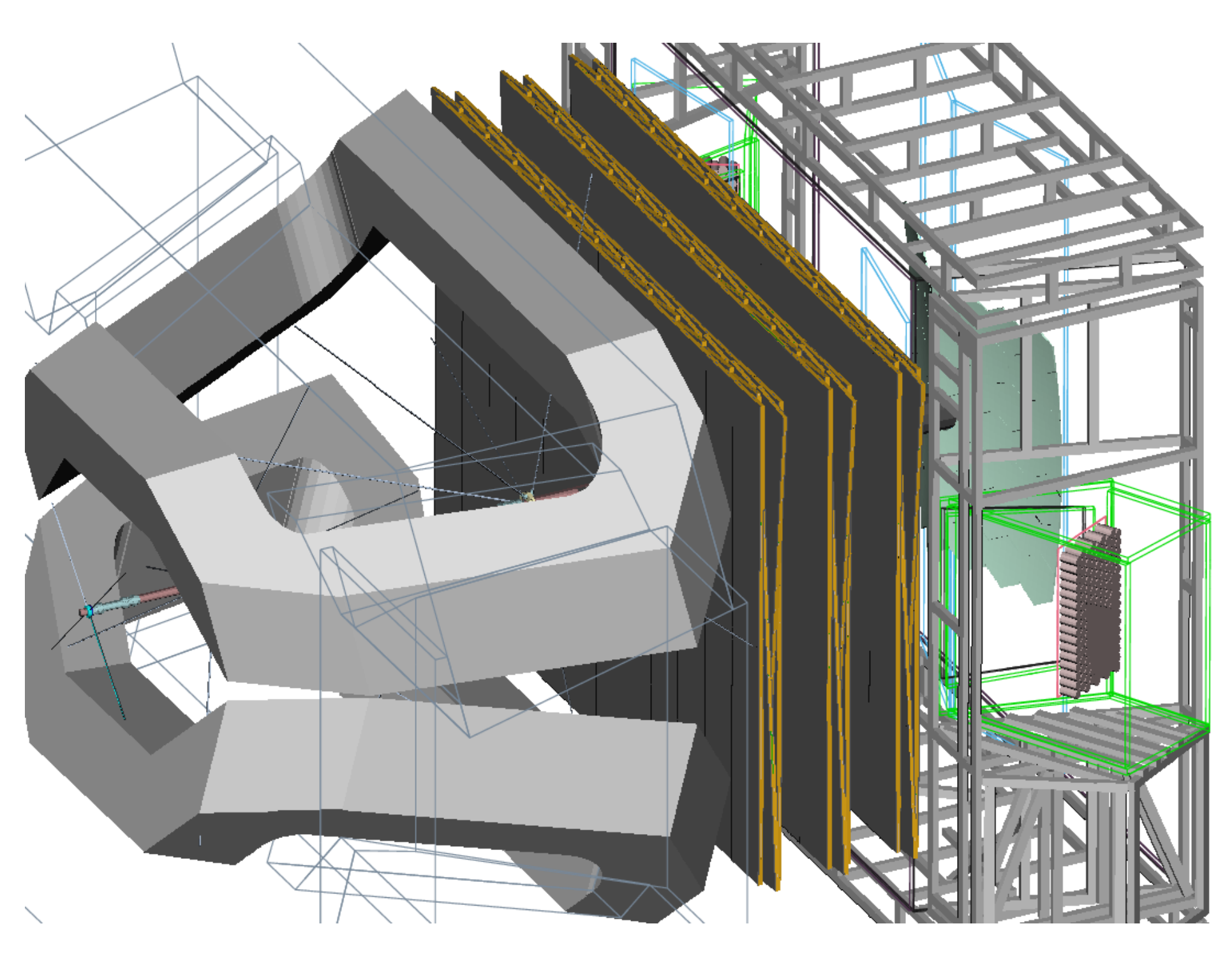

Tracking stations (T1,T2,T3) between magnet (left) and RICH detector (right)

- $\mathrm{LHCb}$ is one of the experiments running at CERN's Large Hadron Collider (LHC).

- SciFi Tracker will upgrade current LHCb experiment on 2018/191.

- Twelve Scintillating Fibres planes of 6x5m readout by Silicon Photo-Multiplier arrays.

\section{SILICON PHOTO-MULTIPLIERS READOUT}

Low Power ASIC for the SCIntillating FIbre traCker (PACIFIC).

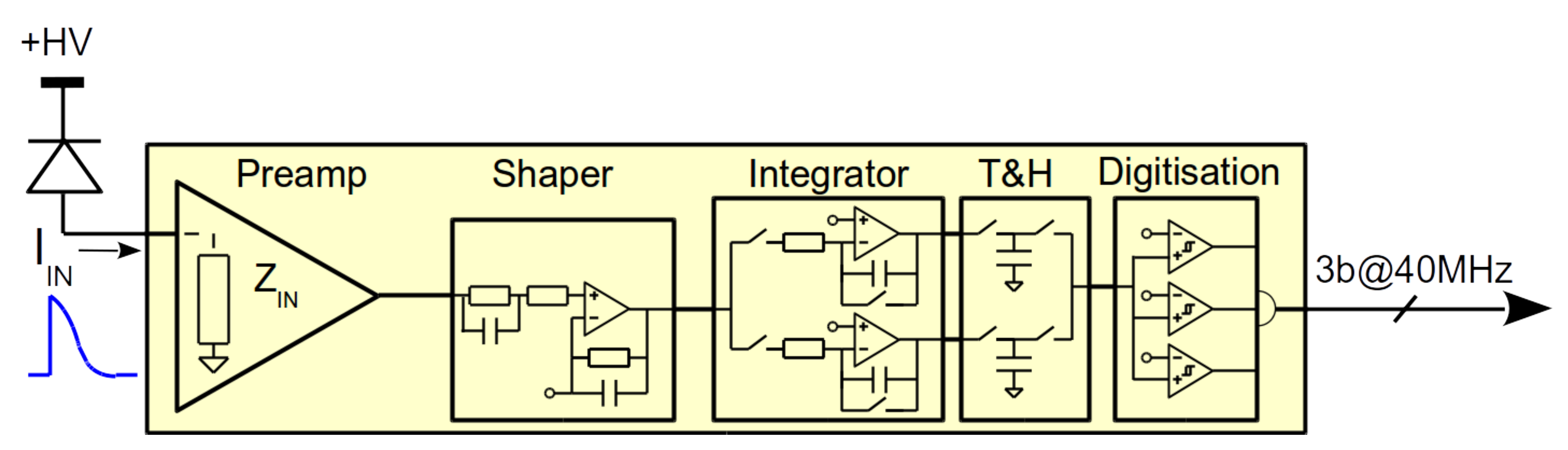

Analog processing chain formed by:

- Current input stage ${ }^{2}$ with transimpedance amplifier.

- Fast Shaper for tail adjustment.

- Double interleaved gated integrator.

- Track and hold (mixing both integrators output).

- Digitization by means of 3 hysteresis comparators.

- Serialization and slow control implemented with standard cells.

\section{FIBRES \& SIPMS}

Detector is formed by scintillating fibre mats;

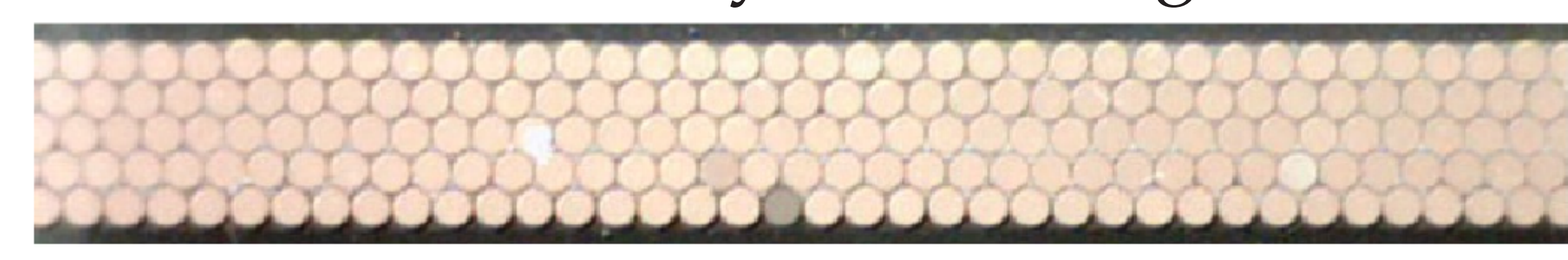

- 5 or 6 layers of stacked fibres.

- $250 \mu \mathrm{m}$ diameter fibres.

Each mat is readout by an array of SiPMs;

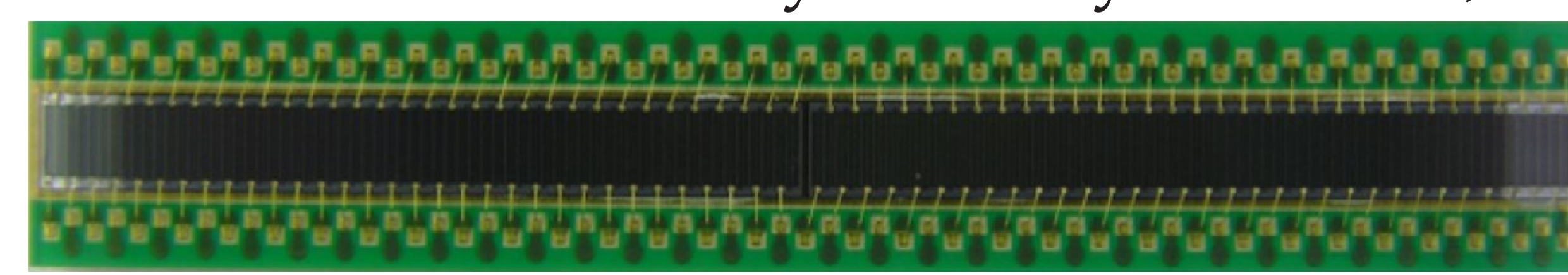

- Two dies of 64 channels form an array.

- Channel pitch is $250 \mu \mathrm{m}$ width.

The fine pitch readout leads to low occupancy and good hit resolution of $<100 \mu \mathrm{m}$.

\section{PACIFIC SPECIFICATIONS}

- 64 channels.

- High Bandwidth ( $\approx 300 \mathrm{MHz})$.

- Low power (<10 mW channel).

- Low input impedance $(\approx 50 \Omega)$.

- Directly connected to SiPM.

- 25ns double peak resolution.

- Fast shaping (tail removal).

- Anode voltage control.

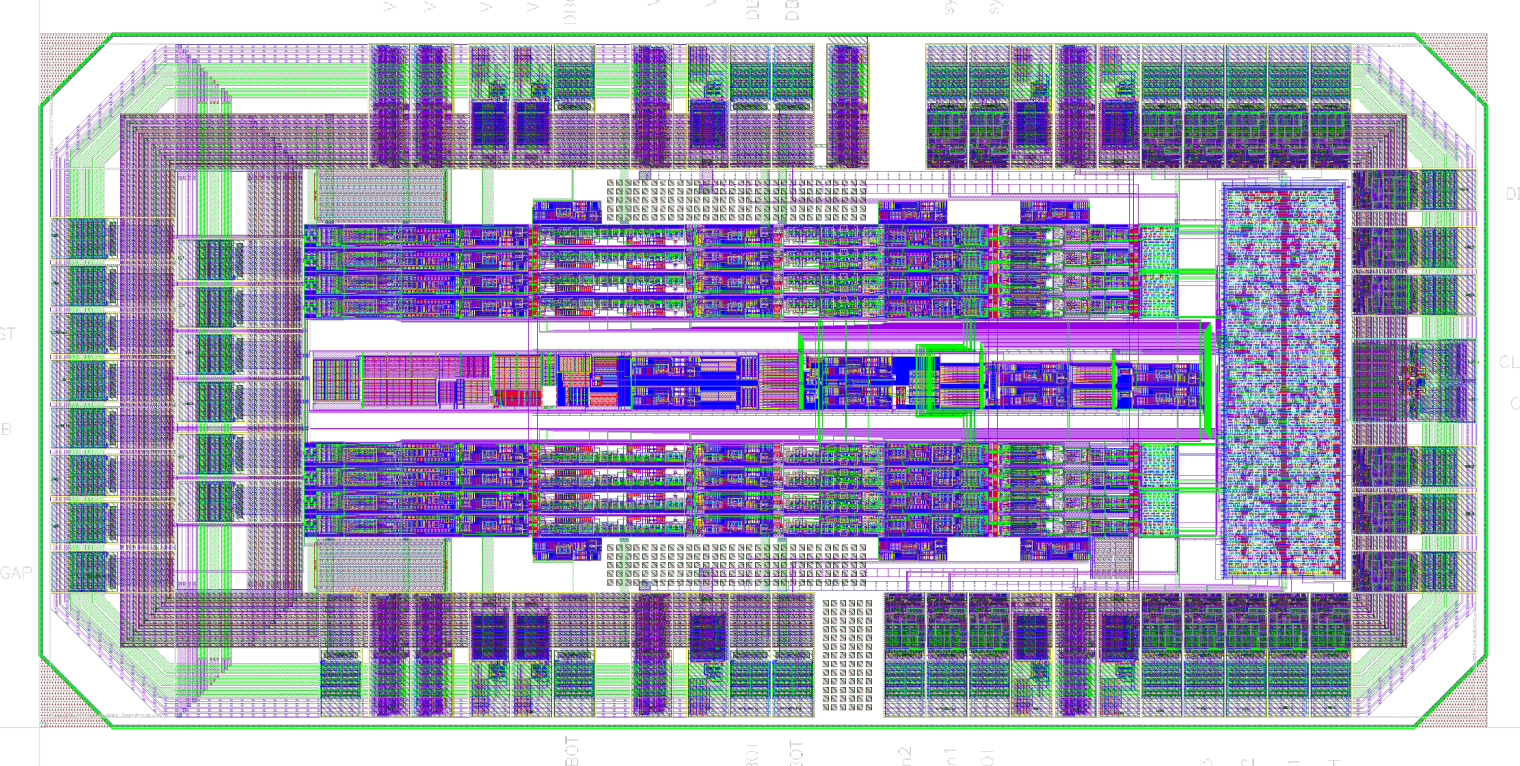

Image of PACIFICr2 produced in IBM 130nm technology including 8 channels.

\section{FIRST PROTOTYPES MEASURES}

Two manufacturers used as baseline solution: Ketek and Hamamatsu with very different signal timing (highly configurable shaper).

Triggering an LED to generate few photons and integrating the output of the shaper during 25ns, photon peaks can be easily measured from next figures.

Input signal simulations from both sensors:

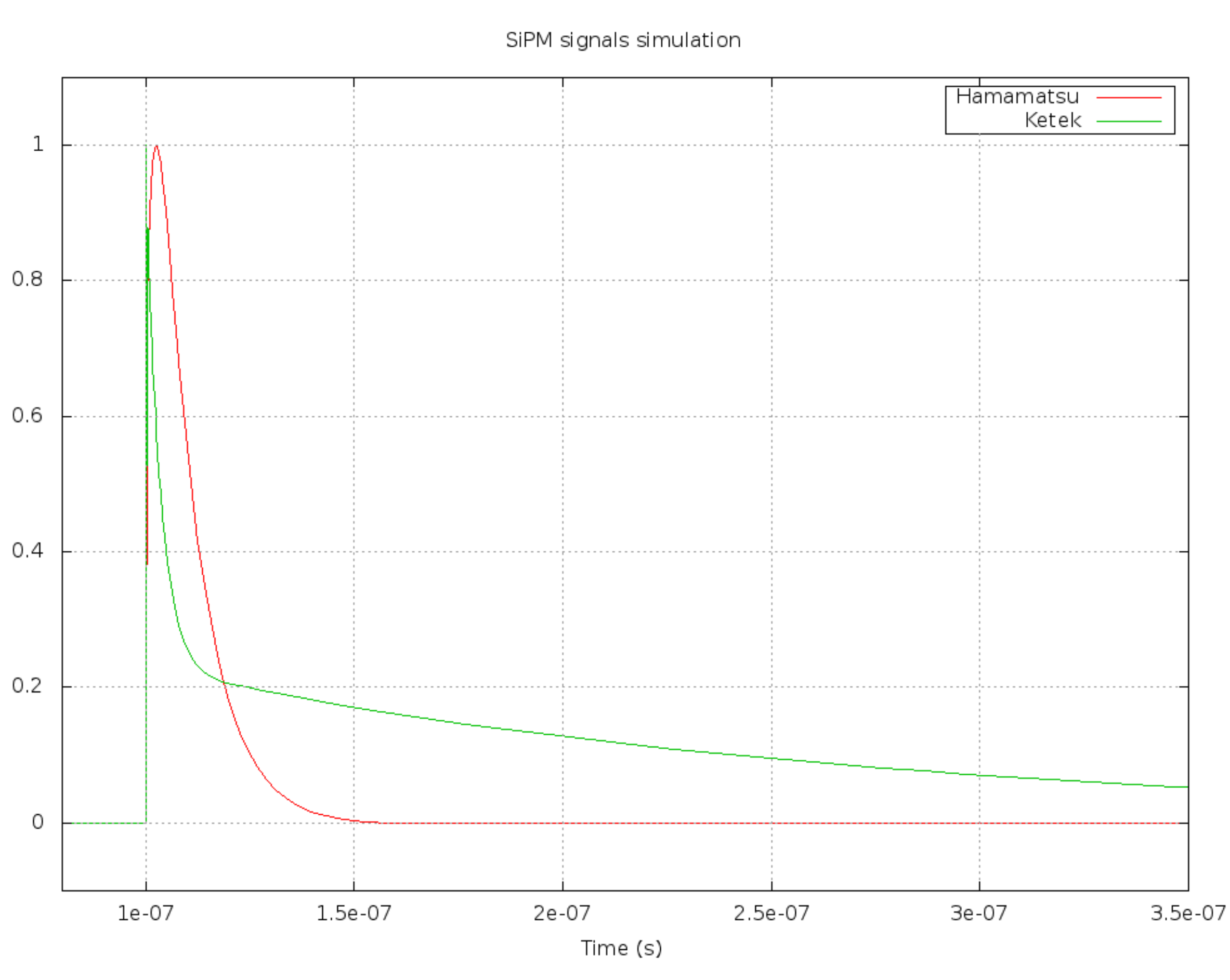

Note different time constants, tail compensation needed and implemented with shaper circuit

\section{REFERENCES}

${ }^{1}$ LHCb Tracker Upgrade Technical Design Report LHCb Collaboration, 2014, LHCB-TDR-015

${ }^{2}$ FlexToT - Current mode ASIC for readout of common cathode SiPM arrays, IEEE NSS/MIC, 2013
Hamamatsu Array measurement:

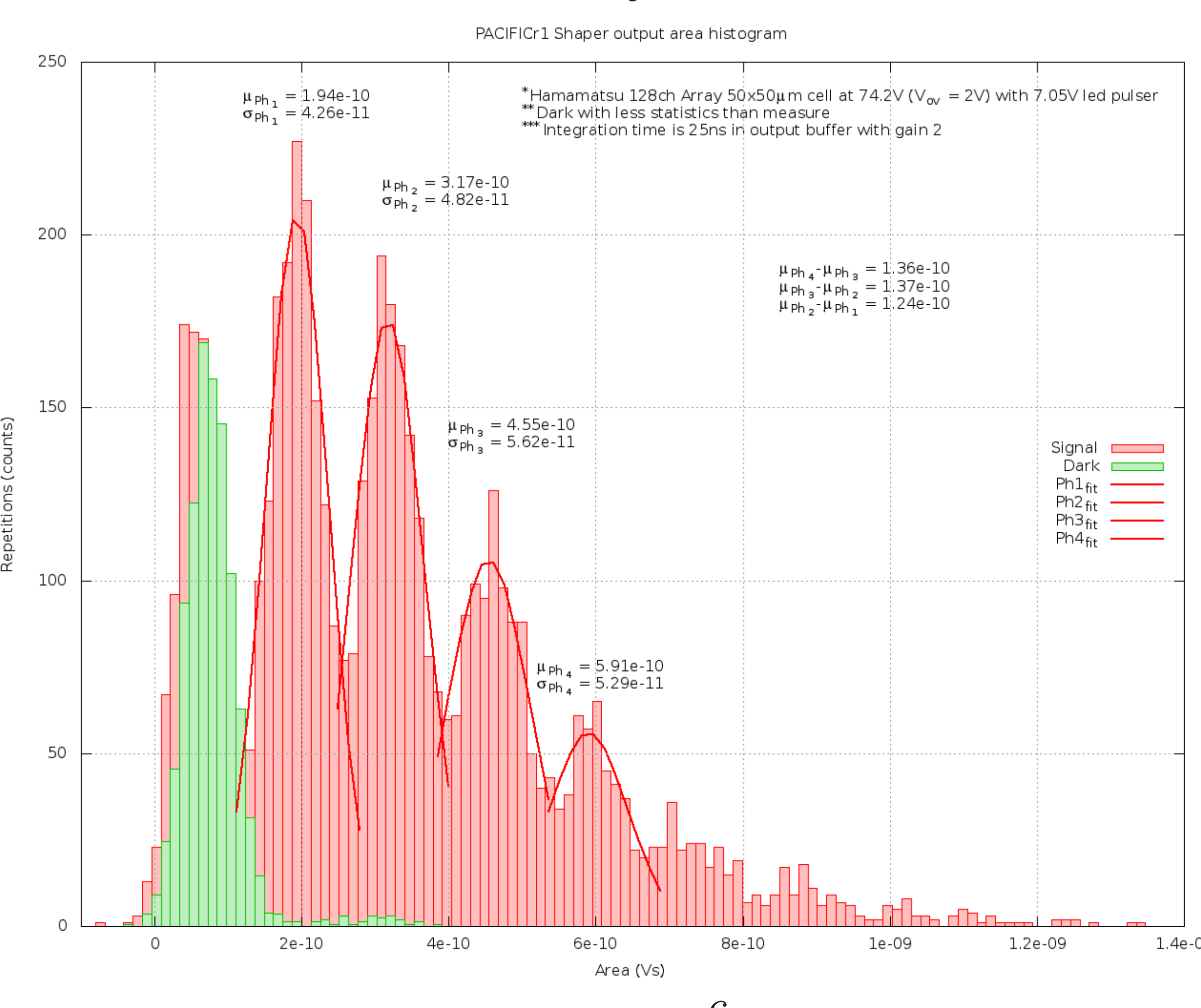

$G \approx 0.89 e^{6}$
Ketek Array measurement:

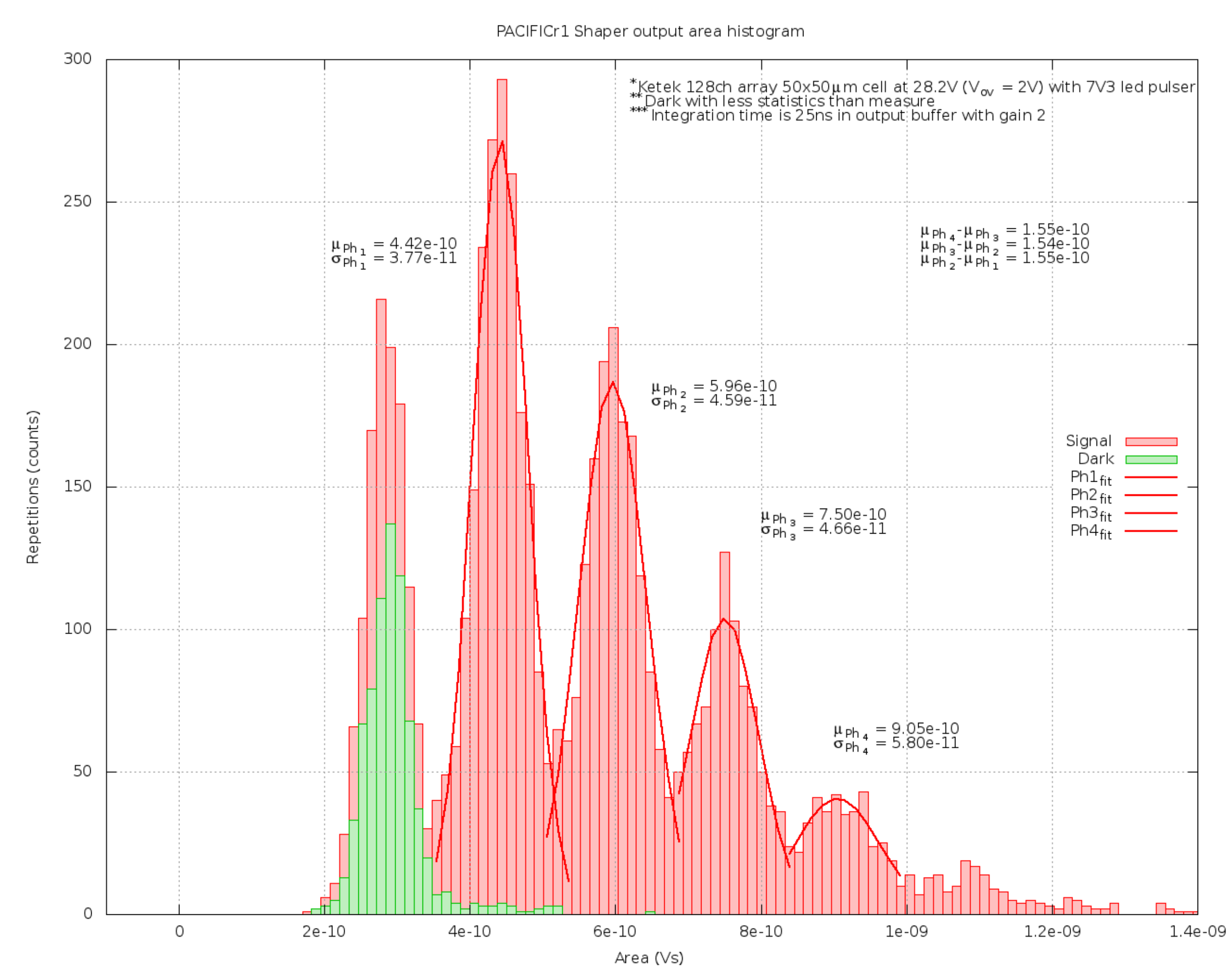

$G \approx 1.1 e^{6}$ 